\title{
¿Azitromicina como tratamiento contra Chlamydia trachomatis?
}

\author{
José Luis Maldonado-Calderón, Francisco Carlos López-Márquez y Pablo Ruiz-Flores
}

Universidad Autónoma de Coahuila, Centro de Investigación Biomédica, Coahuila, México

\begin{abstract}
Resumen
Se ha demostrado la efectividad de la azitromicina y la doxiciclina en el tratamiento de la clamidiasis urogenital, lo que se ha mantenido sin cambios por mucho tiempo. Se ha propuesto la autoinoculación como método de reinfección y persistencia de la enfermedad en las mujeres y también debido a la farmacocinética de la azitromicina en este tejido. Con los nuevos métodos diagnósticos y las pruebas de curación se ha comprobado una diferencia a favor de la doxiciclina en el tratamiento de la clamidiasis rectal. La resistencia antimicrobiana no ha desempeñado un papel relevante porque no se han encontrado cepas resistentes in vivo al tratamiento. A pesar de ello, la azitromicina sigue siendo un fármaco de primera elección ya que puede administrarse como una dosis única, lo que favorece el apego terapéutico.
\end{abstract}

PALABRAS CLAVE: Chlamydia trachomatis. Azitromicina. Prueba de curación.

\begin{abstract}
Azithromycin and doxycycline effectiveness has been demonstrated in the treatment of urogenital chlamydiasis, which has remained unchanged for a long time. Autoinoculation has been proposed as a method of reinfection and persistence of the disease in women and probably also owing to azithromycin pharmacokinetics in this tissue. With the new diagnostic methods and tests of cure, a difference has been demonstrated in favor of doxycycline in the treatment of rectal chlamydiasis Antimicrobial resistance has not played a relevant role since no treatment-resistant strains have been found in vivo. Nevertheless, azithromycin remains a first-choice drug, since it can be administered as a single dose, which favors therapeutic adherence.
\end{abstract}

KEY WORDS: Chlamydia trachomatis. Azithromycin. Test of cure.

\section{Introducción}

La clamidiasis es causada por Chlamydia trachomatis (genotipos A-K), constituye la enfermedad más reportada y se encuentra entre las infecciones de transmisión sexual más prevalentes en Estados Unidos; el grupo etario de 15 a 24 años es el más afectado., ${ }^{1,2}$ De acuerdo con la Organización Mundial de la Salud, anualmente hay un estimado de 131 millones de nuevas infecciones causadas por Chlamydia trachomatis. ${ }^{3}$

La enfermedad es asintomática en $70 \%$ de las mujeres y $50 \%$ de los hombres. ${ }^{4}$ Cuando produce síntomas, las mujeres suelen presentar cambios en la descarga vaginal, sangrado intermenstrual o dispareunia (datos sugestivos de cervicitis); al examen físico se identifica eritema cervical, cérvix friable y descarga endocervical. Además, los síntomas pueden simular infección de vías urinarias. En hombres se manifiesta más comúnmente como uretritis (disuria, molestia y descarga uretral) y al examen físico se observa irritación o eritema del meato urinario y en ocasiones descarga uretral (que tiende a ser mucoide y no purulenta); puede presentarse también en forma de orquitis 0 epididimitis. En mujeres puede causar secuelas graves como enfermedad pélvica inflamatoria, embarazo ectópico, aborto espontáneo e infertilidad.5,6
Correspondencia:

Francisco Carlos López-Márquez

E-mail: francisco.c.lopezmarquez@gmail.com
Fecha de recepción: 09-05-2017

Fecha de aceptación: 30-08-2017

DOI: 10.24875/GMM.17003458
Gac Med Mex. 2018;154:689-692

Disponible en PubMed

www.gacetamedicademexico.com 
Para realizar el diagnóstico de clamidia urogenital se cuenta con diferentes métodos como el cultivo celular, la prueba de diagnóstico rápido, anticuerpos contra clamidia en suero y la prueba de amplificación de ácidos nucleicos (NAAT, nucleic acid amplification test), de elección ya que alcanza la más alta sensibilidad y una especificidad comparable a la del cultivo celular, además, la Organización Mundial de la Salud la recomienda. La muestra idónea para realizar la NAAT en hombres es el primer chorro de la primera orina del día, mientras que en las mujeres es la obtenida mediante frotis vaginal. ,, $6^{-6}$

La Organización Mundial de la Salud y la agencia de los Centers for Disease Control and Prevention (CDC) sugieren como tratamiento de la clamidia $1 \mathrm{~g}$ de azitromicina oral en dosis única o $100 \mathrm{mg}$ de doxiciclina oral dos veces al día por siete días.,7

\section{Azitromicina}

Azitromicina es un antibiótico de la familia de los macrólidos, derivado semisintético de la eritromicina. ${ }^{8}$ Expresa su actividad antibiótica uniéndose a la subunidad 50 S ribosomal (rRNA 23S) e inhibe la síntesis de proteínas al impedir la formación del enlace peptídico entre los aminoácidos de la proteína naciente. ${ }^{9}$ Su perfil farmacocinético está caracterizado por la captura rápida y extensa de la circulación hacia compartimentos intracelulares (incluidas las llamadas células inflamatorias); posteriormente es liberada poco a poco en el sitio donde se necesita, lo que permite un régimen monodosis o dosis por un solo día. ${ }^{9-11}$

\section{¿Azitromicina o doxiciclina?}

Como ya se mencionó, para clamidia urogenital no complicada la Organización Mundial de la Salud y la agencia de los CDC recomiendan $1 \mathrm{~g}$ de azitromicina oral como dosis única o $100 \mathrm{mg}$ de doxiciclina oral dos veces al día por siete días. Esta recomendación está basada en un metaanálisis de 12 ensayos clínicos sobre azitromicina versus doxiciclina para el tratamiento de clamidia urogenital realizado en $2002,{ }^{11}$ el cual demostró que ambas son igualmente eficaces, con tasas de curación microbiana de 97 y $98 \%$ para azitromicina y doxicilina, respectivamente. Sin embargo, en 11 de esos ensayos clínicos se usaron métodos diagnósticos y de seguimiento para curación menos sensibles (cultivo e inmunoensayos) que los actualmente recomendados (NAAT), lo que pudo provocar subestimación de los rangos de falla terapéutica. ${ }^{7,12,13}$ En un nuevo metaanálisis realizado en 2014 se concluyó que existe una eficacia mayor de $3 \%$ a favor de la doxiciclina para el tratamiento de clamidiasis urogenital y de aproximadamente $7 \%$ para el tratamiento de infección uretral sintomática en hombres.13

\section{Clamidiasis rectal}

La diferencia de eficacia entre azitromicina comparada con doxiciclina para el tratamiento de clamidiasis rectal podría ser mayor que la reportada en clamidiasis urogenital. ${ }^{14,15}$ En 2015 se registró una eficacia de $83 \%$ para azitromicina y más de $99 \%$ para doxiciclina en el tratamiento de infección rectal por clamidia, aunque se reconoció que la calidad de la evidencia era baja. ${ }^{16}$ A pesar de esto, una efectividad aproximada de $83 \%$ para azitromicina es mucho menor que $97.4 \%$ indicado en el tratamiento de la clamidiasis urogenital. ${ }^{14}$

Lo anterior podría deberse a la biodisponibilidad de la azitromicina en el tejido rectal, que se desconoce si es similar a la de la mucosa uretral y cervical. Algunos estudios experimentales en animales, que investigan clamidia en el intestino grueso, muestran falta de respuesta inmune local, así como ausencia de células polimorfonucleares ${ }^{17}$ y dado que la azitromicina usa estas células para alcanzar el sitio de infección, la falta de estas y una reacción inmune disminuida en el recto podrían disminuir la efectividad de la azitromicina. En 2013 se examinó la respuesta inflamatoria a la infección rectal por clamidia y se reportaron citocinas inflamatorias suprimidas en sujetos VIH negativos infectados con clamidia, lo cual aporta más datos y sustenta lo mencionado. ${ }^{18}$

Aunado a todo esto, la autoinoculación de clamidia del tracto gastronitestinal hacia el urogenital en mujeres ha sido propuesta y ha tomado relevancia últimamente. Así, el tracto gastrointestinal se ha mencionado como un nicho de infección persistente. ${ }^{17}$ En 2015, mediante un modelo matemático se estimó la probabilidad de que una mujer con infección genital por clamidia tratada, ya fuera con azitromicina o doxiciclina, permaneciera libre de clamidia cuando la posibilidad de autoinoculación era tomada en cuenta; se demostró que cuando se asumía que la probabilidad de autoinoculación era de $100 \%$, la probabilidad de que la paciente permaneciera libre de infección genital después del tratamiento con doxiciclina fue de 96.8 y $81.9 \%$ con azitromicina; esto es, 3.2 y $18.1 \%$ de probabilidades, respectivamente, de no eliminar la infección, 5.7 veces más de no eliminar la infección con azitromicina comparada con doxiciclina. Además, si la paciente presentaba infección 
rectal, la probabilidad de permanecer libre de infección genital después del tratamiento con azitromicina era de 78.2 a $94.3 \%$, y después del tratamiento con doxiciclina de 96.7 a $97.1 \%$, lo cual corresponde de dos a 6.6 veces más probabilidad de no eliminar la infección por clamidia con azitromicina comparada con doxiciclina. ${ }^{15}$

Actualmente, la agencia de los CDC recomienda como tratamiento para clamidia rectal el mismo que para clamidia urogenital, sin embargo, no es más que una extrapolación de los hallazgos sobre la eficacia similar (que se cuestiona) de ambos fármacos en el tratamiento de clamidia urogenital. En un análisis realizado en 2015 en una clínica de enfermedades de transmisión sexual a pacientes masculinos diagnosticados con clamidia rectal entre 1993 y 2012, se encontró que los hombres tratados con azitromicina tuvieron un riesgo significativamente alto de persistencia/recurrencia de clamidia rectal comparados con los sujetos tratados con doxiciclina (resultados similares se obtuvieron en una revisión retrospectiva en $2016^{19}$ ); además, los autores sugieren que un régimen de siete días de doxiciclina puede ser superior a una dosis única de $1 \mathrm{~g}$ de azitromicina para el tratamiento de clamidiasis rectal..$^{20}$ Otro estudio sugiere que $100 \mathrm{mg}$ de doxiciclina dos veces al día son altamente efectivos para clamidiasis rectal asintomática, logrando cura bacteriana (evaluada por NAAT) en $98.8 \%$ de los casos. ${ }^{21}$

\section{Resistencia antimicrobiana}

La resistencia bacteriana a antibióticos ha sido propuesta como causa de falla terapéutica. Dadas las complejidades del ciclo de vida de Chlamydia trachomatis ${ }^{22,23}$ es posible que las formas persistentes, involucradas en resistencia heterotípica, puedan requerir varias semanas para restablecer infección replicativa detectable después del tratamiento con una sola dosis de azitromicina. ${ }^{24}$ Sin embargo, solamente se ha podido documentar resistencia heterotípica (que ocurre cuando una infección tiene una pequeña proporción de organismos resistentes entre una población susceptible) con mutaciones en el rRNA 23S asociadas con resistencia in vitro a macrólidos. A la fecha no han sido identificadas cepas con resistencia homotípica (en la que la población completa de organismos sobrevive después del tratamiento). ${ }^{25,26}$

\section{Prueba de curación falso-positiva}

NAAT es el método de elección para realizar una "prueba de curación". Desafortunadamente este método solamente proporciona información acerca de la presencia de material genético, pero no de la viabilidad del patógeno o si este aún es infeccioso; por lo tanto, los resultados positivos intermitentes pudieran ser el resultado de la deposición de material genético viable o no viable de una pareja sexual, la liberación de material genético de células degradadas o cuerpos elementales que contienen material genético de Chlamydia trachomatis. ${ }^{27}$ Una NAAT negativa puede indicar infección eliminada, sin embargo, también puede reflejar infección persistente. ${ }^{28}$

Actualmente las guías recomiendan una prueba de curación a los tres meses de finalizado el tratamiento, en vez de realizarla a las cuatro semanas de haberlo recibido, para minimizar el riesgo de diagnósticos falso-positivos. ${ }^{25}$ Resultados falso-positivos pueden ocurrir pasadas tres semanas del tratamiento debido a la persistencia de ADN y dado que la enfermedad persistente tarda algunas semanas en emerger. Las pruebas de curación deberían diferirse por al menos cinco semanas postratamiento. ${ }^{22,27}$ Además, se ha demostrado que para evitar la reinfección, las mujeres deberían abstenerse de relaciones sexuales sin condón o, en todo caso, usar condón en cada relación sexual durante al menos un mes después de haber recibido el tratamiento. ${ }^{29}$

\section{Conclusión}

Aun cuando se ha probado que la eficacia en el tratamiento de clamidia urogenital es ligeramente mayor a favor de doxiciclina, la azitromicina sigue siendo un medicamento de elección, ya que se puede administrar como dosis única, alcanzando mayor apego terapéutico. Sin embargo, si se toma en cuenta la autoinoculación del tracto gastrointestinal como causa de falla terapéutica, se debería preferir doxiciclina, puesto que se desconoce la farmacocinética de la azitromicina en el tejido rectal.

Además, la agencia de los CDC recomienda la prueba de curación solamente si la adherencia al tratamiento es cuestionable, si los síntomas persisten o si se sospecha reinfección. No se recomienda su realización si se dio el tratamiento adecuado de la forma adecuada, sin embargo, debido a que la mayoría de las infecciones por clamidia urogenital o rectal son asintomáticas, se ha demostrado mayor eficacia terapéutica contra clamidiasis rectal al usar doxiciclina comparada con azitromicina, si tomamos en cuenta la posibilidad de autoinoculación siempre deberíamos sospechar reinfección, por lo que a toda paciente se le debería practicar una prueba de curación. 
Lo anterior fortalece reconsiderar el tratamiento de clamidia urogenital y tomar en cuenta la infección rectal por clamidia como una fuente de autoinoculación. Además, se aconseja estratificar a las poblaciones en riesgo de padecer clamidia rectal, así como tomar muestras genitales y rectales a pacientes en quienes se sospeche clamidia urogenital.

Es deseable la realización de ensayos controlados aleatorizados comparando la eficacia de azitromicina contra doxiciclina para el tratamiento de clamidia urogenital y rectal.

\section{Bibliografía}

1. Centers for Disease Control and Prevention. Sexually transmitted disease surveillance 2015. EE. UU.: Department of Health and Human Services; 2016.

2. Centers for Disease Control and Prevention. CDC Fact Sheet. Reported STDs in the United States 2015 National Data for Chlamydia, Gonorrhea, and Syphilis. EE. UU.: Department of Health and Human Services; 2016.

3. World Health Organization. Report on global sexually transmitted infection surveillance 2015. Ginebra, Suiza: World Health Organization; 2016.

4. World Health Organization. WHO Guidelines for the Treatment of Chlamydia trachomatis. Ginebra, Suiza: World Health Organization; 2016.

5. Lane AB Decker CF. Chlamydia trachomatis infections. Dis Mon 2016:62:269-273.

6. Ahmadi A, Khodabandehloo M, Ramazanzadeh R, Farhadifar F, Roshani $D$, Ghaderi $E$, et al. The relationship between Chlamydia trachomatis genital infection and spontaneous abortion. J Reprod Infertil. 2016:17:110-116

7. Meyer T. Diagnostic procedures to detect chlamydia trachomatis infections. Microorganisms. 2016;4:25.

8. Workowski KA, Bolan GA, Centers for Disease Control and Prevention. Sexually transmitted diseases treatment guidelines, 2015. MMWR Recomm Rep. 2015;64:1-137.

9. Bakheit A, Al-Hadiya B, Abd-Elgalil A. Azithromycin. En: Brittain $\mathrm{H}$ Profiles of drug substances, excipients and related methodology. EE. UU.: Academic Press; 2014.

10. Cobos-Trigueros N, Ateka O, Pitart C, Vila J. Macrólidos y cetólidos. Enf Infec Microb Clin. 2009;27:412-418.

11. Jelić D, Antolović R. From erythromycin to azithromycin and new potential ribosome-binding antimicrobials. Antibiotics (Basel). 2016;5:29.

12. Lau CY, Qureshi AK. Azithromycin versus doxycycline for genital chlamydial infections: a meta-analysis of randomized clinical trials. Sex Transm Dis. 2002;29:497-502.

13. Geisler W, Uniyal A, Lee J, Lensing S, Johnson S, Perry R et al. Azithromycin versus doxycycline for urogenital chlamydia trachomatis infection. New Engl J Med. 2015;373:2512-2521.
14. Kong FY, Tabrizi SN, Law M, Vodstrcil LA, Chen M, Fairley C, et al. azithromycin versus doxycycline for the treatment of genital chlamydia infection: a meta-analysis of randomized controlled trials. Clin Infect Dis. 2014;59:193-205.

15. Craig A, Kong FY, Yeruva L, Hocking JS, Rank RG, Wilson DP, et al. Is it time to switch to doxycycline from azithromycin for treating genital chlamydial infections in women? Modelling the impact of autoinoculation from the gastrointestinal tract to the genital tract. BMC Infect Dis. 2015;15:200.

16. Kong FY, Tabrizi SN, Fairley CK, Vodstrcil LA, Huston WM, Chen M, et al. The efficacy of azithromycin and doxycycline for the treatment of rectal chlamydia infection: a systematic review and meta-analysis. J Antimicrob Chemother. 2015;70:1290-1297.

17. Rank RG, Yeruva L. Hidden in plain sight: chlamydial gastrointestinal infection and its relevance to persistence in human genital infection. Infect Immun. 2014;82:1362-1371.

18. Heiligenberg $M$, Lutter R, Pajkrt D, Adams K, De-Vries H, Heijman T, et al. Effect of HIV and chlamydia infection on rectal inflammation and cytokine concentrations in men who have sex with men. Clin Vaccine Immunol. 2013;20:1517-1523.

19. Gratrix J, Brandley J, Dane M, Plitt S, Smyczek P, Read R, et al. A retrospective review of treatment failures using azithromycin and doxycycline in the treatment of rectal chlamydia infections in women and men who have sex with Men. Sex Transm Dis. 2016;43:110-112.

20. Khosropour CM, Dombrowski JC, Barbee LA, Manhart L, Golden MR. Comparing azithromycin and doxycycline for the treatment of rectal chlamydial infection: a retrospective cohort study. Sex Transm Dis. 2014;41:79-85.

21. Horner PJ. Azithromycin antimicrobial resistance and genital chlamydia trachomatis infection: duration of therapy may be the key to improving efficacy. Sex Transm Infect. 2012;88(3):154-156.

22. Ziklo N, Huston W, Hocking J, Timms P. Chlamydia trachomatis genital tract infections: when host immune response and the microbiome collide. Trends Microbiol. 2016;24:750-765.

23. Cossé MM, Hayward RD, Subtil A. One face of chlamydia trachomatis: the infectious elementary body. Curr Top Microbiol Immunol. 2018;412:35-58.

24. Elgalib A, Alexander S, Tong C, White JA. Seven days of doxycycline is an effective treatment for asymptomatic rectal chlamydia trachomatis infection. Int J STD AIDS. 2011:22:474-477.

25. Kong FY, Hocking JS. Treatment challenges for urogenital and anorectal chlamydia trachomatis. BMC Infect Dis. 2015;15:293.

26. Madhivanan $\mathrm{P}$, Krupp K. Antibiotic resistance in prevalent bacterial and protozoan sexually transmitted infections. Indian J Sex Transm Dis AIDS. 2015;36:3.

27. Wind C, Schim Van Der Loeff MF, Unemo M, Schuurman R, Van-Dam AP, De-Vries H. Time to clearance of chlamydia trachomatis RNA and DNA after treatment in patients coinfected with Neisseria gonorrhoeae: a prospective cohort study. BMC Infect Dis. 2016;16:554.

28. Dukers-Muijrers N, Morré SA, Speksnijder A, Van Der Sande M, Hoebe C. Chlamydia trachomatis test-of-cure cannot be based on a single highly sensitive laboratory test taken at least 3 weeks after treatment. PLoS One. 2012;7(3):e34108.

29. Lazenby GB, Korte JE, Tillman S, Brown FK, Soper DE. A recommendation for timing of repeat chlamydia trachomatis test following infection and treatment in pregnant and nonpregnant women. Int J STD AIDS. 2016;28:902-909. 